\title{
Antibodies trigger reprogramming
}

Antibodies that initiate signaling cascades and thereby replace reprogramming factors promise an efficient and safe way to produce pluripotent stem cells.

The reversal of time has long intrigued creative minds. The writer F. Scott Fitzgerald explored the challenges of life unfolding in reverse from old age to youth in "The Curious Case of Benjamin Button." In 2006, Shinya Yamanaka from Kyoto University created the cellular equivalent of Benjamin Button when his team brought a fully differentiated mouse fibroblast back to its origin as a pluripotent stem cell.

Yamanaka achieved this reprogramming with the expression of four transcription factors (TFs): Oct4, Sox2, c-Myc and Klf4; many other researchers have since used combinations of these TFs or replaced them with small molecules to change gene expression and induce pluripotency.

As powerful as these current reprogramming methods are, they still suffer from drawbacks - to overexpress TFs, genetic material needs to be introduced, and small molecules can have off-target effects that are not fully understood. Both approaches still have low efficiency. Kristin Baldwin from the Scripps Research Institute saw an opening for an alternative approach. In 2014, her team began a collaboration with Richard Lerner, also at Scripps, to screen for antibodies that could trigger intracellular signaling leading to reprogramming.

Baldwin had two main goals for this work: to better understand biology and to develop a more efficient dedifferentiation method. "We wanted to interrogate reprogramming from the cell surface and identify methods to reprogram without ever going into the nucleus," Baldwin explained. She added, "Antibodies are very easy to evolve and mutate. You can increase their specificity more easily than you can with small molecules."

The team first focused on antibodies that would replace either Sox 2 or Oct4. Mouse embryonic fibroblasts, transfected with the remaining three TFs, were treated with a library of antibodies generated by the Lerner team so that each cell expressed only a few unique antibodies. The researchers then screened for growth of induced pluripotent stem cell (iPSC) colonies, amplified the antibodies in these cells and validated them in a secondary screen.

In both cases the team found candidates, though the efficiencies of Oct4 replacement antibodies were low, and Baldwin decided to pursue the signaling pathway triggered by the antibody that replaced Sox 2 . This led the team to an unexpected result. The antibody bound and antagonized brain acid soluble protein 1 (Basp1), a protein that translocates from the cell membrane to the nucleus and acts as a transcriptional repressor for a tumor-suppressor gene. Basp1 is needed for filopodia formation but has not been implicated in reprogramming or pluripotency. Basp1 repression leads to an upregulation of genes associated with reprogramming, and the team found that directly inhibiting Basp1 mimicked the effect of the antibody.

For Baldwin, the next steps are to optimize the antibodies and try combinations to ideally replace all four reprogramming TFs with antibodies. "We have not been able to get the antibodies to work together," she said. One reason may be the low efficiency of the antibody replacing Oct4; another may be that only certain antibodies work together, and the perturbation of one pathway is not compatible with that of another. A saturating screen of the antibody library with a diversity of $10^{11}$ will likely yield answers, but this also represents a huge amount of work. "My lab can't do everything," Baldwin stated, and she encouraged others to use the approach as well.

It is not just the antibodies that need improving; there is also room for optimization when it comes to choosing the starting cells. Baldwin's team worked with mouse embryonic fibroblasts, but they are thinking of transitioning to human blood cells. Different starting cells will have different cell surface proteins and may require different sets of antibodies.

Ultimately, Baldwin thinks that adding a cocktail of antibodies to the culture medium could turn cells that have so far proven refractory, such as neurons, to reprogramming into an iPSC.

\section{Nicole Rusk}

\section{RESEARCH PAPERS}

Blanchard, J.W. et al. Replacing reprogramming factors with antibodies selected from combinatorial antibody libraries. Nat. Biotechnol. http://dx.doi. org/10.1038/nbt.3963 (2017). 